\title{
Excess body iron and the risk of type 2 diabetes mellitus: a nested case-control in the PREDIMED (PREvention with MEDiterranean Diet) study
}

\author{
Victoria Arija ${ }^{1,2,3 *}$, José C. Fernández-Cao ${ }^{1}$, Josep Basora ${ }^{2,3,4,5}$, Mònica Bulló ${ }^{3,4,5}$, Nuria Aranda ${ }^{1,3}$, \\ Ramón Estruch ${ }^{5,6}$, Miguel A. Martínez-González ${ }^{5,7}$ and Jordi Salas-Salvadó ${ }^{3,4,5 *}$ \\ ${ }^{1}$ Nutrition and Public Health Unit, Universitat Rovira $i$ Virgili, C/Sant Llorenc 21, 43201 Reus, Tarragona, Spain \\ ${ }^{2}$ Reus-Altebrat Primary Care, Institut d'Investigació en Atencio Primària (IDIAP) Jordi Gol, Reus, Spain \\ ${ }^{3}$ Pere Virgili Health Research Institute, Universitat Rovira $i$ Virgili, C/Sant Llorenç 21, 43201 Reus, Tarragona, Spain \\ ${ }^{4}$ Human Nutrition Unit, Universitat Rovira $i$ Virgili, Reus, Tarragona, Spain \\ ${ }^{5}$ CIBERobn Physiopathology of Obesity and Nutrition, Instituto de Salud Carlos III, Madrid, Spain \\ ${ }^{6}$ Department of Internal Medicine, Hospital Clinic, Institut d'Investigació Biomèdica August Pi $i$ Sunyer (IDIBAPS), \\ University of Barcelona, Barcelona, Spain \\ ${ }^{7}$ Department of Preventive Medicine and Public Health, University of Navarra, Pamplona, Spain
}

(Submitted 11 November 2013 - Final revision received 31 July 2014-Accepted 7 August 2014 - First published online 17 October 2014)

\begin{abstract}
A prospective nested case-control study within the PREvention with MEDiterranean Diet (PREDIMED) was conducted to evaluate the relationship between excess body Fe (measured as serum ferritin (SF), soluble transferrin receptor (sTfR) and sTfR:ferritin ratio) and the risk of type 2 diabetes mellitus (T2DM) in a Mediterranean population at a high risk of CVD, without T2DM at the start of the study. The study contained 459 subjects, 153 with incident T2DM (cases) and 306 without incident T2DM (controls). The follow-up period was for 6.0 (interquartile range 3.9-6.5) years. For each incident diabetic subject, two subjects were selected as controls who were matched broadly for age as well as for sex, intervention group and BMI. We observed a relationship between SF values $>257 \mu \mathrm{g} / \mathrm{l}$ in males and $>139 \mu \mathrm{g} / \mathrm{l}$ in females and the risk of T2DM, following adjustment in the conditional logistic regression model for high-sensitivity C-reactive protein, fasting glucose and other components of the metabolic syndrome (OR 3.62, 95\% CI 1.32, 19.95; $P=0.022)$. We also found an association between low sTfR:ferritin ratio levels and the incidence of T2DM (OR 3.02, 95\% CI 1.09, 8.39; $P=0.042$ ), but no association with sTfR (OR $1.29,95 \%$ CI $0.51,3 \cdot 23 ; P=0.722$ ). Oxidative stress has been hypothesised to contribute to the development of insulin resistance and $\beta$-cell dysfunction, the two key events in the clinical development of T2DM. Following adjustment for other risk factors for T2DM, excess body Fe (measured as SF and sTfR:ferritin ratio) was associated with an increased risk of developing T2DM in a Mediterranean population at a high risk of CVD.
\end{abstract}

Key words: Serum ferritin: Soluble transferrin receptor: Body iron stores: Type 2 diabetes

The worldwide prevalence of diabetes in adults was estimated as $6.4 \%$ in 2010 , and has been forecast to increase to $7.7 \%$ by $2030^{(1)}$. Recently, excess body Fe has been shown to be a risk factor for type 2 diabetes mellitus (T2DM) ${ }^{(2)}$.

Serum ferritin (SF) is the most widely used biomarker of body $\mathrm{Fe}$ stores in epidemiological studies, despite being shown to be affected by inflammation status. Conversely, soluble transferrin receptor (sTfR) is not altered by inflammatory processes $^{(3)}$, and their levels in blood are proportional to the cell requirements for $\mathrm{Fe}^{(4)}$.

Several prospective studies ${ }^{(2,5-14)}$ have identified excess $\mathrm{Fe}$ as a risk factor for T2DM. Oxidative stress could be the mechanism by which excess $\mathrm{Fe}$ is associated with a higher incidence of T2DM. Oxidative stress would mediate in the pathophysiology of several key events related to the onset of T2DM, such as insulin resistance (IR) and $\beta$-cell dysfunction $^{(15)}$. Most of these studies ${ }^{(2,5-12)}$ used SF as a biomarker to estimate body Fe levels. However, in assessing the relationships between SF and the risk of T2DM, few studies had adjusted for fasting glucose levels ${ }^{(8)}$ and other components of the metabolic syndrome (MetS) ${ }^{(5)}$, which are the parameters with a high predictive capacity for $\mathrm{T}_{2} \mathrm{DM}^{(16)}$. As such, it is still not clear whether the relationship between SF levels and the risk of $\mathrm{T} 2 \mathrm{DM}$ is independent of these risk factors.

Abbreviations: CLR, conditional logistic regression; hs-CRP, high-sensitivity C-reactive protein; IR, insulin resistance; MetS, metabolic syndrome; PREDIMED, PREvention with MEDiterranean Diet; sTfR, soluble transferrin receptor; SF, serum ferritin; T2DM, type 2 diabetes mellitus.

*Corresponding authors: Dr V. Arija, fax +34 977 759322, email victoria.arija@urv.cat; Dr J. Salas-Salvadó, fax +34 977759322 , email jordi.salas@urv.cat 
Few prospective studies evaluating the association between excess body $\mathrm{Fe}$ and the risk of T2DM have used STfR as a biomarker $^{(2,7,11)}$, and despite having found that elevated SF levels increased the risk of T2DM, the relationship between sTfR and T2DM was not clear. Thus, while the Potsdam European Prospective Investigation into Cancer and Nutrition (EPIC) cohort study did not observe any association ${ }^{(2)}$, the Diabetes Prevention Program (DPP) cohort study ${ }^{(7)}$ observed that high levels of sTfR increased the risk of T2DM. Also, the recent Kuopio IHD Risk Factor (KIHD) study observed a U-shaped association ${ }^{(11)}$.

To date, no studies have been conducted in southern Europe exploring the relationship between excess Fe and the risk of T2DM. Of note is that some characteristics of Fe metabolism, such as genetic predisposition and/or dietary intake in southern Europe, are quite different from the populations studied earlier, such as those in the USA ${ }^{(7)}$, China ${ }^{(10)}$ and northern Europe ${ }^{(2)}$. For example, while in northern Europe, the prevalence of the C282Y polymorphism in the haemochromatosis (HFE) gene is $5-10 \%$ and that of the H63D polymorphism is $10-20 \%$, in southern Europe, the prevalence is $1-5 \%$ and $>20 \%$, respectively $^{(17)}$. In Spain, the prevalence of the H63D mutation reaches $46 \%$ in certain regions ${ }^{(18)}$. Furthermore, the consumption of food items of animal origin is greater in northern compared with southern Europe ${ }^{(19)}$.

To test the hypothesis that high body Fe stores increase the risk of T2DM in our geographical area, we measured excess body Fe (as SF, sTfR and sTfR:ferritin ratio) in relation to the risk of $\mathrm{T} 2 \mathrm{DM}$ in a Mediterranean population at a high risk of $\mathrm{CVD}$, without $\mathrm{T} 2 \mathrm{DM}$ at the start of the prospective study.

\section{Experimental methods}

\section{Study design}

This is a case-control study nested in the PREDIMED (PREvention with MEDiterranean Diet) cohort, followed-up for a median of 6.0 (interquartile range 3.9-6.5) years. The PREDIMED trial ${ }^{(20)}$ was intended to test the effectiveness of the Mediterranean diet on the primary prevention of CVD. The comparisons were between two traditional Mediterranean diets (one enriched with extra virgin olive oil and the other with nuts) $v$. advice alone on a low-fat diet. The present study was registered at ClinicalTrials.gov (registration no. ISRCTN35739639; http://www.controlledtrials. com/ISRCTN35739639).

The present study was conducted according to the guidelines laid down in the Declaration of Helsinki, and all procedures involving human subjects were approved by the Clinical Research Ethics Committee of the Hospital Sant Joan de Reus. Written informed consent was obtained from all subjects.

\section{Subjects}

The present study was conducted with 459 Caucasian subjects aged 55-80 years, free of T2DM at baseline, and with three or more CVD risk factors; 153 were incident T2DM (cases) and 306 non-incident (control) individuals. The study population was recruited in the Primary Care Centres of Reus, Barcelona and Pamplona. For every incident diabetic individual identified, two subjects were randomly selected as controls matched for age ( $\leq 67 v .>67$ years), sex, intervention group and BMI $\left(\leq 27 v .>27 \mathrm{~kg} / \mathrm{m}^{2}\right)$, using an incidence density sampling procedure.

\section{Ascertainment of incident type 2 diabetes mellitus}

The 153 cases of incident T2DM were diagnosed during the follow-up, according to the American Diabetes Association criteria $^{(21)}$, i.e. fasting plasma glucose concentration $\geq 7 \cdot 0 \mathrm{mmol} / 1$ or plasma glucose concentration $\geq 11 \cdot 1 \mathrm{mmol} / 1$ measured $2 \mathrm{~h}$ after a $75 \mathrm{~g}$ oral glucose load. A routine glucose test was performed on all participants in the PREDIMED study at least once a year to detect new cases of diabetes. When new-onset T2DM was identified by the physicians of Primary Care Centres, the test was repeated within the next 3 months to confirm the diagnosis. The homeostasis model assessment (HOMA) index was calculated for each individual as follows:

$$
\begin{aligned}
\text { HOMA-IR }= & \text { fasting insulin }(\mathrm{U} / \mathrm{l}) \\
& \times \text { fasting glucose }(\mathrm{mmol} / \mathrm{l}) / 22 \cdot 5) .
\end{aligned}
$$

\section{Biochemical determination}

All blood samples were collected after an overnight fast at the beginning of the study. Aliquots of serum and EDTA plasma were immediately processed, coded and shipped to a central laboratory in a portable cooler $\left(-4^{\circ} \mathrm{C}\right)$, and stored at $-80^{\circ} \mathrm{C}$ until analysis. The time between blood sampling and freezing was less than $1 \mathrm{~h}$. Serum levels of fasting glucose, total TAG, total and HDL-cholesterol were measured by standard enzymatic methods. LDL-cholesterol was calculated using the Friedewald equation. Fasting plasma insulin concentrations were measured in duplicate by ELISA (Ezhi-14K; Millipore). Plasma concentrations of high-sensitivity C-reactive protein (hs-CRP) were measured using a highly sensitive immunoassay (Helica Biosystems, Inc.). The assay has a sensitivity of $0.2 \mu \mathrm{g} / 1$, with intra- and inter-assay $\mathrm{CV}$ of $\leq 3.7$ and $<4.8 \%$, respectively. SF (Elecsys Ferritin; Roche Diagnostics) and sTfR (Access sTfR 0QC; Beckman Coulter) were measured by immunochemiluminescence. The assay has a sensitivity of $0.05 \mu \mathrm{g} / 1$ for ferritin, and intra- and inter-assay CV of $\leq 2$ and $<3.5 \%$, respectively, for $\mathrm{SF}$. The assay has a sensitivity of $0.05 \mathrm{nmol} / \mathrm{l}$ for sTfR, and intra- and inter-assay CV of $\leq 5$ and $\leq 8 \%$, respectively $(1 \mathrm{mg} / \mathrm{l}=13.55 \mathrm{nmol} / 1$ of $\mathrm{sTfR})$.

\section{Other measures}

At baseline and at each annual visit, a general questionnaire on sociodemographic and lifestyle characteristics was administered, and anthropometric variables were measured. Also, a semi-quantitative 137 -item FFQ that had been previously validated $^{(22)}$ was applied. Nutrients and energy intake were quantified according to the Spanish food composition

a $55-80$ years, free of T2DM at baseline, and with three or 
tables ${ }^{(23)}$. Leisure-time physical activity was assessed according to a validated questionnaire ${ }^{(24)}$. Blood pressure was measured in triplicate using a calibrated semi-automatic oscillometer (Omron HEM-705CP; Omron Healthcare Europe BV) ${ }^{(25)}$.

\section{Statistical methods}

Variables showing a non-normality of distribution were log-transformed to normalise the distributions. Qualitative variables were compared using the $\chi^{2}$ test. Quantitative variables were compared using the Student's $t$ test or the Mann-Whitney test. Data are presented as percentages, means or geometric means and standard deviations, and medians and interquartile ranges in the case of variables being non-normally distributed.

Partial correlation coefficients of SF, sTfR and the sTfR:ferritin ratio adjusted for sex, age and BMI, as well as for several T2DM risk factors such as MetS components, fasting insulin, HOMA-IR and hs-CRP were calculated in the overall study sample, controls and cases (Table 2). A multiple linear regression analysis was applied to evaluate the influence of SF on IR.

To analyse the relationship between body Fe levels and the incidence of T2DM, participants were categorised into quartiles according to the distributions of SF, sTfR and STfR:ferritin ratio at baseline in control individuals. Given the documented differences between males and females with respect to body Fe stores, the independent variables were adjusted for sex. The adjusted variables were categorised to avoid any assumption of linearity, and to evaluate the dose-response relationship in the onset of T2DM. Several conditional logistic regression (CLR) models were applied. A crude model (without adjustment) was fitted with each independent variable. The model was re-fitted with adjustment for lifestyle variables including the following: marital status (married/not married); educational level (primary/secondary/tertiary); smoking (current smoker/former smoker/never smoked); alcohol consumption (drinker/non-drinker); physical activity (200 metabolic equivalents (MET)-min/d/ $\geq 200 \mathrm{MET}-\mathrm{min} / \mathrm{d}$ ); family history of T2DM (yes/no). Adjustment also included four diagnostic criteria of the MetS, according to the harmonised criteria proposed by International Diabetes Federation (IDF) and the American Heart Association/National Heart, Lung, and Blood Institute (AHA/NHLBI) ${ }^{(26)}$, i.e. blood pressure $\geq 135 / 85 \mathrm{mmHg}$, serum TAG $\geq 1.7 \mathrm{mmol} / 1$, HDL-cholesterol $<1.03 \mathrm{mmol} / \mathrm{l}$ in males and $<1.3 \mathrm{mmol} / \mathrm{l}$ in females, and waist circumference $\geq 102 \mathrm{~cm}$ in men and $\geq 88 \mathrm{~cm}$ in women. Dietary variables measured in relation to the risk of T2DM were categorised by quartiles, and included energy, $\mathrm{Mg}$, vitamins $\mathrm{D}$ and $\mathrm{E}$, dairy products, meat, vegetables and fruits $^{(27)}$. Then, hs-CRP (mg/l) was introduced in model 1. Finally, fasting glucose concentration $\geq 5.6 \mathrm{mmol} / \mathrm{l}$ was introduced into the model. The test for linear trend across the quartiles was performed by assigning the median value to each category and introducing these new variables into the CLR as continuous variables.

The effects of SF and fasting glucose on the risk of T2DM were evaluated in another CLR model adjusted as 'enter' mode for all the variables included in model 1 , except SF and fasting glucose, which were introduced as 'conditional' mode (CLR-conditional). We performed the same analysis for sTfR and the sTfR:ferritin ratio.

All data analyses were performed with the SPSS package for Windows (version 20.0; SPSS, Inc.). A value of $P<0.05$ was considered statistically significant.

\section{Results}

Of the 459 subjects, two individuals were removed from the study for not having the values of SF and STfR recorded, and another two for having extreme values of SF. The final analysis contained 455 individuals.

There were no significant interactions between SF, sTfR and the sTfR:ferritin ratio $v$. sex $(P>0 \cdot 05)$. These interactions were assessed in the same CLR models in which we studied the association between high SF, STfR and the sTfR:ferritin ratio levels and the risk of T2DM.

Table 1 summarises the baseline characteristics of the overall group, as well as the case and control groups separately. Of the participants, $71 \%$ had abdominal obesity, $42 \%$ the MetS, 34\% hypertriacylglycerolaemia, 97\% hypertension and $25 \%$ IR. Additionally, prevalences of the MetS and IR were higher in the cases than in the controls. Compared with the controls, patients with incident diabetes had greater waist circumference and higher levels of fasting glucose, insulin, TAG and IR. Also, SF levels were higher in the cases than in the controls, while sTfR levels were similar in both groups.

Table 2 summarises the partial correlation coefficients adjusted for sex, age and BMI between SF, STfR and the sTfR:ferritin ratio together and several T2DM risk factors such as MetS components, fasting insulin, HOMA-IR and hsCRP in the overall study sample, and in the controls as well as cases. SF and sTfR:ferritin ratio, but not sTfR levels, correlated significantly with fasting glucose, fasting insulin and HOMA-IR in the overall study sample. Furthermore, both biomarkers of body Fe stores significantly correlated with fasting insulin and HOMA-IR in non-incident diabetic individuals, and with SF alone in incident diabetic subjects. Conversely, SF was not correlated with hs-CRP, while sTfR was positively correlated with this biomarker of inflammation. SF and the sTfR: ferritin ratio were poorly correlated with the components of the MetS. Nevertheless, components such as blood pressure, TAG and HDL-cholesterol were significantly correlated with sTfR in the overall non-incident diabetic group.

To evaluate the association between the levels of SF and the risk of T2DM, several CLR models were applied (Table 3). The OR for the crude model (without adjustment for any variable) was 1.99 (95\% CI 1.12, 3.52; $P=0.022$ ). Model 1 adjusted for lifestyle variables, family history of T2DM, and four components of the MetS showed an improvement in the trend (OR 2.39, 95\% CI 1.11, 5.16; $P=0.030$ ). This trend did not substantially change when hs-CRP was included in the multivariable model (OR 2.38, $95 \%$ CI 1.10, 5.14; $P=0 \cdot 031$ ). Finally, adjusting the model for fasting glucose showed an improvement in the trend (OR 3.62, $95 \%$ CI 1.32, 9.95; $P=0.017$ ).

The same models were employed in evaluating the relationship between the sTfR:ferritin ratio and the risk of T2DM. 
Table 1. Baseline characteristics of the overall study participants, and segregated by cases and controls (Mean values and standard deviations; medians and interquartile ranges (IQR))

\begin{tabular}{|c|c|c|c|c|c|c|c|}
\hline & \multicolumn{2}{|c|}{ All $(n 455)$} & \multicolumn{2}{|c|}{$\begin{array}{c}\text { Non-incident } \\
\text { diabetics }(n \text { 302) }\end{array}$} & \multicolumn{2}{|c|}{$\begin{array}{l}\text { Incident diabetics } \\
\quad(n \text { 153) }\end{array}$} & \multirow[b]{2}{*}{$P$} \\
\hline & Mean & SD & Mean & SD & Mean & SD & \\
\hline \multicolumn{8}{|l|}{ Sociodemographic characteristics } \\
\hline Men (\%) & \multicolumn{2}{|c|}{$47 \cdot 0$} & \multicolumn{2}{|c|}{$47 \cdot 1$} & \multicolumn{2}{|c|}{$47 \cdot 2$} & 1.000 \\
\hline Age (years) & $66 \cdot 30$ & 6.04 & $66 \cdot 29$ & $6 \cdot 12$ & $66 \cdot 33$ & $5 \cdot 89$ & 0.940 \\
\hline Married (\%) & \multicolumn{2}{|c|}{78.50} & \multicolumn{2}{|c|}{79.50} & \multicolumn{2}{|c|}{76.50} & 0.462 \\
\hline Low educational level (\%) & \multicolumn{2}{|c|}{69.70} & \multicolumn{2}{|c|}{69.90} & \multicolumn{2}{|c|}{69.30} & 0.898 \\
\hline Family history of diabetes (\%) & \multicolumn{2}{|c|}{$28 \cdot 10$} & \multicolumn{2}{|c|}{$24 \cdot 20$} & \multicolumn{2}{|c|}{35.90} & 0.008 \\
\hline Lifestyle & & & & & & & \\
\hline Never smoked (\%) & & & & & & & \\
\hline Current smoker (\%) & & & & & & & 0.239 \\
\hline Former smoker (\%) & & & & & & & \\
\hline Alcohol consumer $(\%)$ & & & & & & & 0.657 \\
\hline Sedentary behaviour (\%) & & & & & & & 0.359 \\
\hline Anthropometry & & & & & & & \\
\hline Weight (kg) & $77 \cdot 27$ & 10.97 & 76.65 & $10 \cdot 79$ & 78.46 & 11.25 & 0.091 \\
\hline BMI $\left(\mathrm{kg} / \mathrm{m}^{2}\right)$ & 29.91 & 3.06 & 29.77 & 2.99 & $30 \cdot 20$ & $3 \cdot 17$ & 0.157 \\
\hline Waist circumference $(\mathrm{cm})$ & 99.59 & $9 \cdot 79$ & 98.50 & $9 \cdot 79$ & $101 \cdot 73$ & 9.46 & 0.001 \\
\hline Obesity (\%) & & & & & & & 0.588 \\
\hline Diet & & & & & & & \\
\hline Energy $(\mathrm{kJ} / \mathrm{d})$ & 9923.77 & 2463.45 & 9884.81 & $2362 \cdot 25$ & $10000 \cdot 67$ & $2658 \cdot 32$ & 0.636 \\
\hline Dairy products (g/d) & $357 \cdot 70$ & 218.66 & 359.56 & $212 \cdot 18$ & 354.03 & 231.59 & 0.799 \\
\hline Meat $(\mathrm{g} / \mathrm{d})$ & 139.01 & $52 \cdot 32$ & 138.63 & $53 \cdot 25$ & 139.75 & $50 \cdot 60$ & 0.830 \\
\hline Vegetables $(\mathrm{g} / \mathrm{d})$ & 284.41 & 109.90 & $280 \cdot 60$ & $104 \cdot 10$ & 291.92 & $120 \cdot 55$ & 0.323 \\
\hline Fruits $(g / d)$ & 344.19 & 184.49 & 344.65 & 183.04 & 343.27 & 187.93 & 0.940 \\
\hline Alcohol $(\mathrm{g} / \mathrm{d})$ & 13.00 & 18.03 & 11.98 & $17 \cdot 14$ & $15 \cdot 02$ & 19.55 & 0.105 \\
\hline $\mathrm{Mg}(\mathrm{mg})$ & 363.08 & 83.82 & $361 \cdot 20$ & 79.40 & $366 \cdot 79$ & $92 \cdot 10$ & 0.523 \\
\hline Vitamin D $(\mathrm{mg} / \mathrm{d})$ & 5.69 & $3 \cdot 20$ & $5 \cdot 77$ & 3.22 & 5.54 & $3 \cdot 18$ & 0.474 \\
\hline Vitamin $E(\mathrm{mg} / \mathrm{d})$ & 9.92 & 3.63 & $9 \cdot 81$ & 3.40 & $10 \cdot 14$ & 4.05 & 0.369 \\
\hline $\mathrm{Fe}(\mathrm{mg} / \mathrm{d})$ & $16 \cdot 13$ & 3.77 & $16 \cdot 02$ & 3.55 & $16 \cdot 35$ & $4 \cdot 17$ & 0.403 \\
\hline $\mathrm{Haem} \mathrm{Fe}(\mathrm{mg} / \mathrm{d})$ & 1.47 & 0.58 & 1.44 & 0.58 & 1.52 & 0.58 & 0.138 \\
\hline Biochemistry & & & & & & & \\
\hline Total cholesterol $(\mathrm{mmol} / \mathrm{l})$ & $5 \cdot 80$ & 0.97 & $5 \cdot 84$ & 0.91 & 5.73 & 1.08 & 0.251 \\
\hline LDL-cholesterol (mmol/l) & & & & & & & 0.161 \\
\hline Median & & & & & & & \\
\hline IQR & 3.03 & 1.20 & 3.0 & 4.23 & 2.97 & & \\
\hline HDL-cholesterol $(\mathrm{mmol} / \mathrm{l})$ & $1.39^{*}$ & 0.03 & $1.41^{*}$ & 0.03 & $1.34^{*}$ & 0.03 & 0.030 \\
\hline $\mathrm{TAG}(\mathrm{mmol} / \mathrm{l})$ & $1.45^{*}$ & 0.02 & $1.41^{*}$ & 0.02 & $1.54^{*}$ & 0.02 & 0.031 \\
\hline Fasting glucose $(\mathrm{mmol} / \mathrm{l})$ & & & & & & & $<0.001$ \\
\hline Median & & & & & & & \\
\hline IQR & 4.9 & .83 & & 5.49 & $5 \cdot 55$ & & \\
\hline Fasting insulin (pmol/l) & $33.45^{*}$ & $1 \cdot 80$ & $32 \cdot 00^{*}$ & 1.79 & $36 \cdot 54^{\star}$ & 1.86 & 0.022 \\
\hline HOMA-IR & $1 \cdot 14^{\star}$ & 1.88 & $1.03^{\star}$ & 1.83 & $1.40^{*}$ & 1.89 & $<0.001$ \\
\hline $\mathrm{SF}(\mu \mathrm{g} / \mathrm{l})$ & $112 \cdot 27^{\star}$ & 2.53 & $105 \cdot 22^{*}$ & 2.53 & $127 \cdot 59^{\star}$ & 2.53 & 0.037 \\
\hline $\mathrm{sTfR}(\mathrm{mg} / \mathrm{l})$ & $1.27^{\star}$ & 1.27 & $1 \cdot 27^{\star}$ & $1 \cdot 26$ & $1 \cdot 26^{*}$ & 1.29 & 0.930 \\
\hline sTfR:ferritin ratio & $11 \cdot 27^{*}$ & $2 \cdot 81$ & $12 \cdot 03^{*}$ & $2 \cdot 80$ & $9 \cdot 9^{*}$ & $2 \cdot 80$ & 0.057 \\
\hline hs-CRP (mg/l) & & & & & & & 0.756 \\
\hline Median & & & & & & & \\
\hline IQR & 0.33 & 1.01 & 0.3 & $4 \cdot 12$ & $0 \cdot 30$ & & \\
\hline Metabolic syndrome (\%) & & & & & & & $<0.001$ \\
\hline High waist circumference (\%)† & & & & & & & $<0.001$ \\
\hline Low HDL-cholesterol (\%)‡ & & & & & & & 0.129 \\
\hline HTG (\%)§ & & & & & & & 0.013 \\
\hline $\mathrm{HT}(\%) \|$ & & & & & & & 0.867 \\
\hline High fasting glucose (\%)q & & & & & & & $<0.001$ \\
\hline Insulin resistance $(\%)$ & & & & & & & $<0.001$ \\
\hline 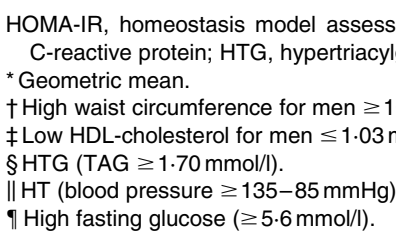 & $\begin{array}{l}t \text { for insulin } \\
\text { rolaemia; } \\
m \text { and wome } \\
\text { I/l and wome }\end{array}$ & $\begin{array}{l}\text { istance; SF } \\
\text { yypertensior } \\
=88 \mathrm{~cm} . \\
=1.30 \mathrm{mmol}\end{array}$ & um fe & R, sol & errin & $\mathrm{As}-\mathrm{CF}$ & tivi \\
\hline
\end{tabular}


In the crude model, low levels of the sTfR:ferritin ratio showed a significant trend towards increased incidence of T2DM (OR $1.73,95 \%$ CI $0.99,3.05 ; P=0.042$ ). In model 1 the trend increased (OR $2.32,95 \%$ CI 1.08, 4.98; $P=0.035$ ). The introduction of hs-CRP did not alter the relationship very much (OR $2 \cdot 31,95 \%$ CI $1 \cdot 08,4 \cdot 97 ; P=0 \cdot 036$ ). Finally, following the inclusion of glucose as a potential confounding variable, the association increased considerably (OR 3.02, 95\% CI 1.09 , 8.39; $P=0 \cdot 42$ ).

In contrast to SF and the sTfR:ferritin ratio, no significant association was observed between sTfR and T2DM after applying the same models. The corresponding multivariate OR for the lowest $v$. highest quartile of sTfR was 1.29 (95\% CI $0.51,3 \cdot 23 ; P=0 \cdot 722$ ).

When we introduced $\mathrm{SF}$ and fasting glucose as 'conditional' mode (CLR-conditional), we observed that fasting glucose was the strongest predictor of diabetes (OR 20.07, 95\% CI 8.36, $48.20 ; P<0.001)$ along with SF (OR 3.62, $95 \%$ CI 1.32, 9.95; $P<0.017)$. In the model of sTfR, the OR of glucose was 16.58 (95\% CI $7 \cdot 15,38.41 ; P<0.001)$ and of sTfR was 1.29 (95\% CI $0.51,3.23 ; P<0.722$ ). Finally, in the sTfR:ferritin ratio model, the OR of glucose was 17.51 (95\% CI 7.50 , $40.89 ; P<0.001)$ and of the sTfR:ferritin ratio was $3.02(95 \%$ CI 1.09, 8.39; $P<0.042$ ).

\section{Discussion}

In the present study, a direct relationship was demonstrated between high body Fe stores (measured as SF and sTfR:ferritin ratio) and the incidence of T2DM in a Mediterranean cohort with an elevated risk of CVD. This association was found after adjustment for hs-CRP, fasting glucose and other components of the MetS. These findings add data from population that is different from those previously studied, i.e. a southern European population. We did not observe any association between the levels of sTfR and the incidence of T2DM.

The prospective design of the study helps reduce temporality bias. Also, the study design enables a better control of confounding factors such as sex and ranges of age and BMI since each case in the incident T2DM group was broadly matched for these variables with two control individuals without T2DM.

SF and sTfR were measured using immunochemiluminescence, a widely used method with high sensitivity and specificity. The diagnosis of T2DM was according to the criteria of the reference organisation, i.e. the American Diabetes Association $^{(21)}$. Of note is that, in the present study, not only was there an adjustment made for classical variables predictive of T2DM risk (such as age, family history of T2DM, smoking, dietary intake, waist circumference and inflammation), but also adjustment for fasting glucose and other components of the MetS since these components are strongly associated with the development of $\mathrm{T}_{2} \mathrm{DM}^{(28)}$.

We need to highlight the limitation of extrapolating the results of the present study to the general population, given that the study was conducted in a population with various CVD risk factors. As occurs in cohort studies, there is no 
Table 3. Risk of type 2 diabetes segregated by quartiles of serum ferritin (SF), soluble transferrin receptor (sTfR) and sTfR:ferritin ratio, and adjusted for sex

(Odds ratios and $95 \%$ confidence intervals)

\begin{tabular}{|c|c|c|c|c|c|c|c|c|}
\hline \multirow{2}{*}{$\overline{\mathrm{SF}}$} & \multirow[t]{2}{*}{ Q1 } & \multicolumn{2}{|r|}{ Q2 } & \multicolumn{2}{|r|}{ Q3 } & \multicolumn{2}{|r|}{ Q4 } & $P_{\text {trend }}$ \\
\hline & & & & & & & & \\
\hline Men $(\mu \mathrm{g} / \mathrm{l})$ & $<78.80$ & \multicolumn{2}{|c|}{$78 \cdot 80-161 \cdot 60$} & \multicolumn{2}{|c|}{$161.60-256.90$} & \multicolumn{2}{|r|}{$>256.90$} & \\
\hline Women $(\mu \mathrm{g} / \mathrm{l})$ & $<48 \cdot 13$ & \multicolumn{2}{|c|}{$48 \cdot 13-96 \cdot 27$} & \multicolumn{2}{|c|}{$96 \cdot 27-139 \cdot 00$} & \multicolumn{2}{|r|}{$>139.00$} & \\
\hline Cases & 30 & \multicolumn{2}{|c|}{36} & & 31 & \multicolumn{2}{|r|}{56} & \\
\hline Controls & 75 & \multicolumn{2}{|r|}{76} & & 76 & \multicolumn{2}{|r|}{75} & \\
\hline Crude* $^{*}$ & 1 & 1.25 & $0.68,2.31$ & 1.06 & $0.57,1.96$ & 1.99 & $1 \cdot 12,3.52$ & 0.022 \\
\hline Model 1† & 1 & 1.09 & $0.50,2.40$ & 0.76 & $0.35,1.67$ & 2.39 & $1 \cdot 11,5 \cdot 16$ & 0.030 \\
\hline Model $1+$ hs-CRP $\ddagger$ & 1 & 1.09 & $0.49,2.39$ & 0.75 & $0.34,1.66$ & $2 \cdot 38$ & $1 \cdot 10,5 \cdot 14$ & 0.031 \\
\hline \multirow[t]{2}{*}{ Model $1+$ hs-CRP $\ddagger$ glucose§ } & 1 & $1 \cdot 17$ & $0.44,3.07$ & 0.65 & $0.24,1.79$ & 3.62 & $1.32,9.95$ & 0.017 \\
\hline & Q4 & \multicolumn{2}{|l|}{ Q3 } & \multicolumn{2}{|l|}{ Q2 } & \multicolumn{2}{|l|}{ Q1 } & \\
\hline \multicolumn{9}{|l|}{ sTfR } \\
\hline Men (mg/l) & $>1.45$ & \multirow{2}{*}{\multicolumn{2}{|c|}{$\begin{array}{l}1.22-1.45 \\
1.27-1.46\end{array}$}} & \multicolumn{2}{|c|}{$1.07-1.22$} & \multicolumn{2}{|r|}{$<1.07$} & \\
\hline Women (mg/l) & $>1.46$ & & & \multicolumn{2}{|c|}{$1.09-1.27$} & \multicolumn{2}{|r|}{$<1.09$} & \\
\hline Cases & 36 & \multicolumn{2}{|c|}{39} & & 36 & \multirow{2}{*}{\multicolumn{2}{|c|}{$\begin{array}{l}42 \\
74\end{array}$}} & \\
\hline Controls & 76 & \multicolumn{2}{|r|}{76} & & 76 & & & \\
\hline Crude $^{*}$ & 1 & $1 \cdot 11$ & $0.63,1.97$ & 1.02 & $0.59,1.77$ & $1 \cdot 21$ & $0.70,2.09$ & 0.499 \\
\hline Model $1 \dagger$ & 1 & 1.76 & $0.82,3.78$ & $1 \cdot 10$ & $0.55,2.21$ & 1.39 & $0.68,2.87$ & 0.575 \\
\hline Model $1+$ hs-CRP $\ddagger$ & 1 & 1.76 & $0.82,3.79$ & $1 \cdot 11$ & $0.55,2.22$ & 1.39 & $0.68,2.86$ & 0.583 \\
\hline \multirow[t]{2}{*}{ Model $1+$ hs-CRP $\ddagger$ glucose $\S$} & 1 & 1.59 & $0.61,4.18$ & $1 \cdot 14$ & $0.47,2 \cdot 76$ & 1.29 & $0.51,3.23$ & 0.722 \\
\hline & Q4 & Q3 & & Q2 & & Q1 & & \\
\hline sTfR:ferritin ratio & & & & & & & & \\
\hline Men $(\mu \mathrm{g} / \mathrm{l})$ & $>15.04$ & & $9-15 \cdot 04$ & & $5-7 \cdot 29$ & & $<4.35$ & \\
\hline Women $(\mu \mathrm{g} / \mathrm{l})$ & $>25.41$ & & $2-25 \cdot 41$ & & $3-14.02$ & & $<8.53$ & \\
\hline Cases & 32 & & 33 & & 34 & & 54 & \\
\hline Controls & 75 & & 76 & & 76 & & 75 & \\
\hline Crude* & 1 & 1.04 & $0.56,1.93$ & $1 \cdot 10$ & $0.61,1.97$ & 1.73 & $0.99,3.05$ & 0.042 \\
\hline Model $1 \dagger$ & 1 & 1.39 & $0.61,3.14$ & 1.04 & $0.48,2.22$ & 2.32 & $1.08,4.98$ & 0.035 \\
\hline Model $1+$ hs-CRP $\ddagger$ & 1 & 1.38 & $0.61,3.13$ & 1.03 & $0.48,2 \cdot 21$ & $2 \cdot 31$ & $1.08,4.97$ & 0.036 \\
\hline Model $1+$ hs-CRP $\ddagger+$ glucose $\S$ & 1 & 1.65 & $0.58,4.67$ & 1.06 & $0.40,2.79$ & 3.02 & $1.09,8.39$ & 0.042 \\
\hline
\end{tabular}

Q, quartile; hs-CRP, high-sensitivity C-reactive protein.

${ }^{*}$ Crude: unadjusted.

† Model 1: marital status (married/not married); educational level (low/medium/high); smoking status (current/former/never); physical activity ( $<200 \mathrm{metabolic}$ equivalents (MET)-min/d or $\geq 200 \mathrm{MET}-\mathrm{min} / \mathrm{d}$ ); alcohol consumption (yes/no); family history of diabetes (yes/no); waist circumference (men <102 or $\geq 102 \mathrm{~cm}$, women $<88$ or $\geq 88 \mathrm{~cm})$; hypertension $(<135$, 85 or $\geq 135 / 85 \mathrm{mmHg})$; hypertriacylglycerolaemia $(<1.70 \mathrm{or} \geq 1.70 \mathrm{mmol} / \mathrm{l})$; HDL-cholesterol (men $\geq 1.03$ or $<1.03 \mathrm{mmol} / \mathrm{l}$, women $\geq 1.30$ or $<1.30 \mathrm{mmol} / \mathrm{l}$ ); diet (energy, dairy products, meat, vegetables, fruits, Mg, and vitamins $\mathrm{D}$ and $\mathrm{E}$ ).

$\ddagger$ hs-CRP in $\mathrm{mg} / \mathrm{l}$.

$\S$ Glucose $<5.6$ or $\geq 5.6 \mathrm{mmol} / \mathrm{l}$

assurance that some of the control individuals would not develop T2DM subsequent to the follow-up period. However, the median period of follow-up of $6 \cdot 0$ (interquartile range $3.9-6.5)$ years is greater than that in the majority of studies conducted to date ${ }^{(2,5-7,10,13)}$

SF, which closely reflects the estimation of body $\mathrm{Fe}$ levels, has been observed to be influenced by inflammation status. Hence, hs-CRP was measured in the present study. The objective was to adjust for this confounding variable when evaluating the effect of excess Fe in relation to T2DM.

Also, we assessed the relationship of another marker that measures Fe status, i.e. sTfR. sTfR in plasma is directly proportional to the cell requirements for $\mathrm{Fe}^{(4)}$. Hence, it is considered to represent a good biomarker in the evaluation of body Fe status ${ }^{(3)}$. However, some studies have suggested that sTfR levels are increased by other factors such as the degree of glucose tolerance or $\operatorname{IR}^{(29)}$, hyperinsulinaemia ${ }^{(30)}$, inflammation $^{(31)}$, general obesity and/or abdominal obesity ${ }^{(32)}$. Also, the sTfR:ferritin ratio appears to be a better marker of Fe stores ${ }^{(33)}$. It is especially useful in population studies since it is sensitive not only to elevated, but also decreased, levels of Fe stores ${ }^{(34)}$.
In the present results, we observed significantly higher levels of ferritin (127.59 (SD 2.53) v. 105.22 (SD 2.53) $\mu \mathrm{g} / \mathrm{l})$ and lower levels of sTfR:ferritin ratio (9.9) (SD 2.80) v. 12.03 (SD 2.80)) with borderline significance in subjects with incident diabetes compared with those with non-incident diabetes. However, we did not observe any difference in sTfR levels. When analysing the relationship using adjusted CLR models, we observed that the values of SF $>257 \mu \mathrm{g} / \mathrm{l}$ in males and $>139 \mu \mathrm{g} / \mathrm{l}$ in females were associated with a high risk of the appearance of T2DM. These values of SF are typical of Fe overload status, according to the WHO $(>200 \mu \mathrm{g} / \mathrm{l}$ in males and $>150 \mu \mathrm{g} / 1$ in females) ${ }^{(35)}$, and are similar to the values found in the studies of Norfolk ${ }^{(6)}$ and Potsdam ${ }^{(2)}$ of the EPIC cohort and the Atherosclerosis Risk in Communities (ARIC) study ${ }^{(5)}$ conducted in the general population.

The associations encountered between SF and T2DM were maintained following the adjustment for classic risk factors including inflammation, fasting glucose and other components of the MetS. The MetS, and its components, have been strongly associated with the development of T2DM, especially with an elevated level of fasting glucose ${ }^{(28)}$. Many prospective studies have adjusted their models using some of these classic 
risk factors, including inflammation ${ }^{(2,5-7,9-11)}$. However, the associations, following the adjustment for fasting glucose and other components of the MetS, have not been observed previously. Only the ARIC study ${ }^{(5)}$, conducted with US males and females between 45 and 64 years of age, observed the association, and which was lost when adjusted for the components of the MetS. Another study, conducted with cohorts from the Finish FINRISK and Health $2000^{(8)}$ studies composed of males and females $>25$ years of age, encountered increased SF levels related to the incidence of T2DM even following the adjustment for glucose, but not for the components of the MetS. In the present study, SF and fasting glucose entered the CLR-conditional model significantly, suggesting that the two variables are risk factors for T2DM. This is in concordance with the current consensus that glucose is the principal risk factor for $\mathrm{T} 2 \mathrm{DM}$, which can indicate that, apart from glucose, SF plays an aetiologic role in the development of this pathology. Another study performed in the general adult population have as well observed SF and glucose as the risk factors of $\mathrm{T} 2 \mathrm{DM}^{(8)}$.

As we have stated above, the sTfR:ferritin ratio seems to be a good biomarker of $\mathrm{Fe}$ deposits given that it is sensitive to elevated as well as decreased levels of $\mathrm{Fe}^{(34)}$. However, it is possible that, since we did not find any association between sTfR and T2DM, the association encountered between the sTfR:ferritin ratio and T2DM could be due exclusively to SF concentrations. Further support for this hypothesis comes from the observation of a strong partial correlation (adjusted for sex, age and BMI) between SF and the sTfR:ferritin ratio, but not so strong a correlation between sTfR and the sTfR: ferritin ratio (Table 2).

The present results and those of previous studies ${ }^{(2-14)}$ suggest that the observed association between ferritin and the sTfR:ferritin ratio $v$. T2DM was very probably due to the excess levels of Fe. Ferritin, in addition to reflecting body $\mathrm{Fe}$ status, increases with inflammation. We measured hs-CRP concentrations to control for this effect in the multivariate analyses of the relationship between excess Fe and the onset of T2DM. In the present results, we observed that hs-CRP levels were similar in incident and non-incident diabetic individuals, while SF was significantly higher in the incident diabetic group. This supports the hypothesis that excess Fe acts independently of the level of inflammation in the development of T2DM. Further support for this interpretation comes from previous studies that have analysed the relationship between ferritin and T2DM following adjustments for one $e^{(2,7,9,10)}$ or more ${ }^{(6)}$ inflammatory markers showing similar findings to ours.

In the $\mathrm{DPP}^{(7)}$ cohort of obese subjects with impaired basal glucose, an association was found not only between SF and T2DM, but also between STfR and T2DM. This latter relationship was contrary to expectation, i.e. increased levels of sTfR, indicative of low Fe stores, were associated with an increased risk of T2DM. More recently, the Finnish KIHD cohort ${ }^{(11)}$, conducted with middle-aged men, also observed that $\mathrm{Fe}$ deficiency and excess Fe (using sTfR as a biomarker) increased the risk of T2DM. A recent review has concluded that extreme conditions of $\mathrm{Fe}$ deficiency, as well as $\mathrm{Fe}$ overload, were associated with increased risk of $\mathrm{CVD}^{(36)}$. As such, the hypothesis is that Fe deficiency could also cause an increase in the incidence of T2DM.

Of considerable note as well is that sTfR levels were much higher in those cohorts in whom an association was observed between sTfR and T2DM, e.g. DPP ${ }^{(7)}$ (median 4th quartile $4.4 \mathrm{mg} / \mathrm{l}$; mean of the overall sample $3.4 \mathrm{mg} / \mathrm{l})$ and KIHD ${ }^{(11)}$ $(0.6-8.2 \mathrm{mg} / \mathrm{l})$ studies compared with the present study $(0.69-2.65 \mathrm{mg} / \mathrm{l})$ and the EPIC Potsdam study ${ }^{(2)}$ (mean of the overall sample $1.13 \mathrm{mg} / \mathrm{l}$ ), albeit Fe stores measured by SF were similar in these four cohort studies. Also, sTfR levels have been documented to be affected by mechanisms other than those related to Fe metabolism (such as insulin sensitivity and obesity), and they could be causally linked to T2DM ${ }^{(4)}$. As such, not only lower Fe storage would lead to increased levels of sTfR, but also sTfR would be a biomarker of another factor causally related to the risk of T2DM.

The essential role of ferritin in the organism is in the storage of Fe. Fe is a catalyst for oxygen reactive species and, as such, contributes to oxidative stress ${ }^{(37)}$. A proposed underlying mechanism is that certain 'trigger' molecules associated with some pathologies could open up the structure of the ferritin molecule, thus provoking the liberation of the stored $\mathrm{Fe}^{(37)}$. This, in turn, could favour the risk associated with oxidative stress and its consequences. Experimental data and clinical studies have suggested that an oxidative environment contributes to the development of $\mathrm{IR}^{(38)}$ and $\beta$-cell dysfunction, which are two key events in the clinical development of $\mathrm{T}_{2} \mathrm{DM}^{(15)}$. Also, the increase in oxidative stress provokes an increase in $\beta$-cell apoptosis in studies with animal models ${ }^{(39)}$ and an increase in IR in human in vivo studies ${ }^{(40)}$. Similarly, evidence exists from cross-sectional studies ${ }^{(41)}$ that elevated levels of SF are associated with increased IR. We observed this relationship in the present study $(\beta=0.001 ; P=0.020)$, i.e. for each $\mu \mathrm{g} / \mathrm{l}$ of $\mathrm{SF}$, there was an increase of 0.001 in HOMA-IR. Also, ferritin was correlated with HOMA-IR in the overall study sample, as well as in incident and non-incident diabetic individuals. This suggests that this relationship with HOMA-IR could be occurring in the entire general population. The mechanisms that underlie this relationship have not been identified to date, although there has been speculation that the pro-oxidant role of Fe would activate a series of stress avenues related to the family of serine/threonine kinases and, finally, causing a disruption in the insulin signalling process ${ }^{(38)}$. It is possible then that IR is an intermediate link in the relationship between high Fe deposits and the risk of T2DM.

\section{Conclusion}

Excess body Fe (measured as SF and sTfR:ferritin ratio) is associated with an increased risk of T2DM in a Mediterranean population at a high risk of CVD, even following the adjustment for hs-CRP, fasting glucose and other components of the MetS. This association was not evident with sTfR.

The potential mechanism that mediates this relationship could be related to IR. More studies are warranted to confirm this mechanism since it is becoming increasingly evident that excess $\mathrm{Fe}$ is related to the incidence of T2DM. 


\section{Acknowledgements}

The authors thank the participants for their enthusiastic collaboration, the PREDIMED personnel for excellent logistics assistance, and the personnel of all the affiliated Primary Care Centres of Reus-ICS. Also, we thank the FPU programme of Ministry of Education, Culture and Sports. Editorial assistance was provided by Dr Peter R. Turner (http://Tscimed.com).

The study was funded in part by the Spanish Ministry of Health (Instituto de Salud Carlos III; PI1001407, PI1301090, FIS PI10/0082, G03/140, RD06/0045), the FEDER (Fondo Europeo de Desarrollo Regional), the Public Health Division of the Department of Health of the Autonomous Government of Catalonia, and Caixa Tarragona (10-1343). The Fundación Patrimonio Comunal Olivarero and Hojiblanca SA (Málaga, Spain), California Walnut Commission (Sacramento, CA), Borges SA (Reus, Spain) and Morella Nuts SA (Reus, Spain) donated the olive oil, walnuts, almonds and hazelnuts, respectively, used in the PREDIMED study. None of the funding sources played any role in the design, collection, analysis or interpretation of the data or in the decision to submit the manuscript for publication. CIBER de Obesidad y Nutrición is a national initiative of the Instituto de Salud Carlos III. No funding body had any role in the design, analysis or writing of this article.

The authors' responsibilities are as follows: V. A. took responsibility for designing the study, directing and performing the statistical analyses, interpreting the results, and drafting of the manuscript; J. C. F.-C. contributed to the statistical analyses, interpretation of the data, and the drafting of the manuscript; J. B. conceived and participated in the design of the PREDIMED study, coordinated the fieldwork, participated in the interpretation of the results, and revised the manuscript; M. B. contributed to the interpretation of the results and revised the manuscript; N. A. coordinated the biochemical analyses, contributed to the interpretation of the results, and revised the manuscript; R. E. and M. A. M.-G. conceived and participated in the design of the PREDIMED study and revised the manuscript; J. S.-S. conceived and participated in the design of the PREDIMED study, participated in the interpretation of the results, and revised the manuscript. All authors read and approved the final manuscript.

J. S.-S. is a non-paid member of the Scientific Advisory Board of the International Nut Council. The other authors have no conflict of interest affecting the conduct, or the reporting of, the work submitted.

\section{References}

1. Shaw JE, Sicree RA \& Zimmet PZ (2010) Global estimates of the prevalence of diabetes for 2010 and 2030. Diabetes Res Clin Pract 87, 4-14.

2. Montonen J, Boeing H, Steffen A, et al. (2012) Body iron stores and risk of type 2 diabetes: results from the European Prospective Investigation into Cancer and Nutrition (EPIC)Potsdam study. Diabetologia 55, 2613-2621.

3. Désidéri-Vaillant C, Galinat H, Sapin-Lory J, et al. (2011) [Serum transferrin receptor in the assessment of iron status]. Transfus Clin Biol 18, 36-39.
4. Speeckaert MM, Speeckaert R \& Delanghe JR (2010) Biological and clinical aspects of soluble transferrin receptor. Crit Rev Clin Lab Sci 47, 213-228.

5. Jehn ML, Guallar E, Clark JM, et al. (2007) A prospective study of plasma ferritin level and incident diabetes: the Atherosclerosis Risk in Communities (ARIC) Study. Am J Epidemiol 165, 1047-1054.

6. Forouhi NG, Harding AH, Allison M, et al. (2007) Elevated serum ferritin levels predict new-onset type 2 diabetes: results from the EPIC-Norfolk prospective study. Diabetologia 50, 949-956.

7. Rajpathak SN, Wylie-Rosett J, Gunter MJ, et al. (2009) Biomarkers of body iron stores and risk of developing type 2 diabetes. Diabetes Obes Metab 11, 472-479.

8. Salomaa V, Havulinna A, Saarela O, et al. (2010) Thirty-one novel biomarkers as predictors for clinically incident diabetes. PLOS ONE 5, e10100.

9. Jiang R, Manson JE, Meigs JB, et al. (2004) Body iron stores in relation to risk of type 2 diabetes in apparently healthy women. JAMA 291, 711-717.

10. Sun L, Zong G, Pan A, et al. (2013) Elevated plasma ferritin is associated with increased incidence of type 2 diabetes in middle-aged and elderly Chinese adults. J Nutr 143, 7-13.

11. Aregbesola A, Voutilainen S, Virtanen JK, et al. (2013) Body iron stores and the risk of type 2 diabetes in middle-aged men. Eur J Endocrinol 169, 247-253.

12. Guo X, Zhou D, An P, et al. (2013) Associations between serum hepcidin, ferritin and $\mathrm{Hb}$ concentrations and type 2 diabetes risks in a Han Chinese population. Br J Nutr 110, 2180-2185.

13. Salonen JT, Tuomainen TP, Nyyssönen K, et al. (1998) Relation between iron stores and non-insulin dependent diabetes in men: case-control study. BMJ 317, 727.

14. Le TD, Bae S, Ed Hsu C, et al. (2008) Effects of cardiorespiratory fitness on serum ferritin concentration and incidence of type 2 diabetes: evidence from the Aerobics Center Longitudinal Study (ACLS). Rev Diabet Stud 5, 245-252.

15. Swaminathan S, Fonseca V, Alam M, et al. (2007) The role of iron in diabetes and its complications. Diabetes Care 30 , 1926-1933.

16. Buijsse B, Simmons RK, Griffin SJ, et al. (2011) Risk assessment tools for identifying individuals at risk of developing type 2 diabetes. Epidemiol Rev 33, 46-62.

17. Kucinskas L, Juzenas S, Sventoraityte J, et al. (2012) Prevalence of $\mathrm{C} 282 \mathrm{Y}, \mathrm{H} 63 \mathrm{D}$, and $\mathrm{S} 65 \mathrm{C}$ mutations in hereditary HFE-hemochromatosis gene in Lithuanian population. Ann Hematol 91, 491-495.

18. Aranda N, Viteri FE, Fernández-Ballart J, et al. (2007) Frequency of the hemochromatosis gene $(H F E) 282 \mathrm{C} \rightarrow \mathrm{Y}$, $63 \mathrm{H} \rightarrow \mathrm{D}$, and $65 \mathrm{~S} \rightarrow \mathrm{C}$ mutations in a general Mediterranean population from Tarragona, Spain. Ann Hematol 86, $17-21$.

19. FAO (2009) FAOSTAT. http://faostat.fao.org (accessed May 2013).

20. Martínez-González MÁ, Corella D, Salas-Salvadó J, et al. (2012) Cohort profile: design and methods of the PREDIMED study. Int J Epidemiol 41, 377-385.

21. American Diabetes Association (2013) Diagnosis and classification of diabetes mellitus. Diabetes Care 36, Suppl. 1, S67-S74

22. Fernández-Ballart JD, Piñol JL, Zazpe I, et al. (2010) Relative validity of a semi-quantitative food-frequency questionnaire in an elderly Mediterranean population of Spain. Br J Nutr 103, 1808-1816. 
23. Mataix J (2003) Tablas de composición de alimentos (Food Composition Tables), 4th ed. [J Mataix and M Manas, editors]. Granada: Universidad de Granada.

24. Elosua R, Marrugat J, Molina L, et al. (1994) Validation of the Minnesota Leisure Time Physical Activity Questionnaire in Spanish men. The MARATHOM Investigators. Am J Epidemiol 139, 1197-1209.

25. Redón J \& Coca A (2003) Guidelines for the diagnosis, evaluation and treatment of hypertension: the point of view of the Spanish Society of Hypertension. Med Clin 121 739-740.

26. Alberti KGMM, Eckel RH, Grundy SM, et al. (2009) Harmonizing the metabolic syndrome. Circulation 120, $1640-1645$.

27. Salas-Salvadó J, Martinez-González MÁ, Bulló M, et al. (2011) The role of diet in the prevention of type 2 diabetes. Nutr Metab Cardiovasc Dis 21, Suppl. 2, B32-B48.

28. Zeng P, Zhu X, Zhang Y, et al. (2011) Metabolic syndrome and the development of type 2 diabetes among professionals living in Beijing, China. Diabetes Res Clin Pract 94, 299-304

29. Fernández-Real JM, Moreno JM, López-Bermejo A, et al. (2007) Circulating soluble transferrin receptor according to glucose tolerance status and insulin sensitivity. Diabetes Care 30, 604-608.

30. Davis RJ, Corvera S \& Czech MP (1986) Insulin stimulates cellular iron uptake and causes the redistribution of intracellular transferrin receptors to the plasma membrane. J Biol Chem 261, 8708-8711.

31. Kasvosve I, Gomo ZAR, Nathoo KJ, et al. (2006) Association of serum transferrin receptor con centration with markers of inflammation in Zimbabwean children. Clin Chim Acta 371, 130-136.

32. Tussing-Humphreys LM, Nemeth E, Fantuzzi G, et al. (2010) Elevated systemic hepcidin and iron depletion in obese premenopausal females. Obesity (Silver Spring) 18, 1449-1456.

33. Malope BI, MacPhail AP, Alberts M, et al. (2001) The ratio of serum transferrin receptor and serum ferritin in the diagnosis of iron status. BrJ Haematol 115, 84-89.

34. Skikne BS (2008) Serum transferrin receptor. Am J Hematol 83, 872-875.

35. World Health Organization (2011) Serum ferritin concentrations for the assessment of iron status and iron deficiency in populations. http://www.who.int/vmnis/indicators/serum_ ferritin.pdf (accessed May 2013).

36. Lapice E, Masulli M \& Vaccaro O (2013) Iron deficiency and cardiovascular disease: an updated review of the evidence. Curr Atheroscler Rep 15, 358.

37. Watt RK (2011) The many faces of the octahedral ferritin protein. Biometals 24, 489-500.

38. Rains JL \& Jain SK (2011) Oxidative stress, insulin signaling, and diabetes. Free Radic Biol Med 50, 567-575.

39. Bertelsen M, Anggård EE \& Carrier MJ (2001) Oxidative stress impairs insulin internalization in endothelial cells in vitro. Diabetologia 44, 605-613.

40. Cooksey RC, Jouihan HA, Ajioka RS, et al. (2004) Oxidative stress, $\beta$-cell apoptosis, and decreased insulin secretory capacity in mouse models of hemochromatosis. Endocrinology 145, 5305-5312.

41. Pham NM, Nanri A, Yi S, et al. (2013) Serum ferritin is associated with markers of insulin resistance in Japanese men but not in women. Metabolism 62, 561-567. 